A

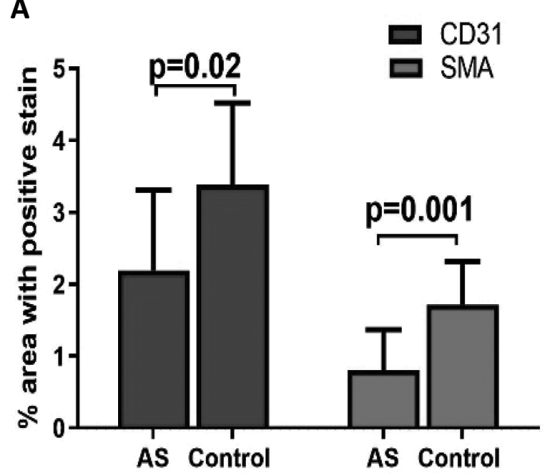

B

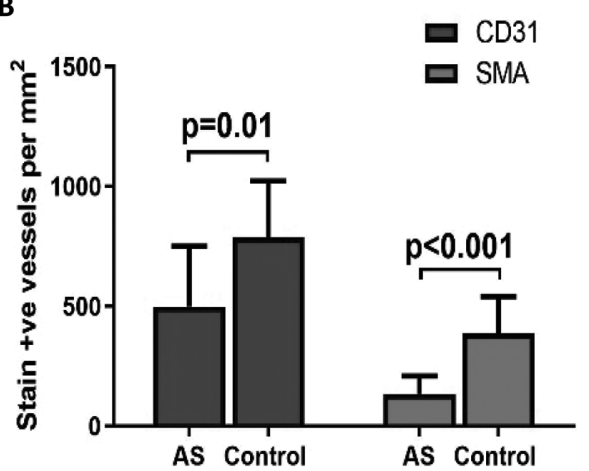

Abstract 122 Figure 1 (A) Reduction in quantity of endothelium (CD31) and smooth muscle actin (SMA) in severe aortic stenosis, (B) reduction in positively stained vessel in severe aortic stenosis

Conclusion In severe AS, there is microvascular rarefaction and loss of endothelium, which is more pronounced in patients with the most severe aortic valve narrowing. This appears to be an underlying mechanism for reduced myocardial perfusion reserve, which may be reversible post AVR.

Conflict of Interest None

\section{THE IMPACT OF PANEL SIZE ON THE YIELD OF GENETIC TESTING IN HYPERTROPHIC CARDIOMYOPATHY: A SYSTEMATIC REVIEW}

${ }^{1}$ Ellie Quinn, ${ }^{1}$ Dee Howley*, ${ }^{1}$ Constantinos O'Mahony, ${ }^{1}$ Mohammed Majid Akhtar, ${ }^{1}$ Konstantinos Savvatis, 'Luis Lopes, ${ }^{1}$ Paula Funnell. 'Barts Heart Centre; ${ }^{2}$ Queen Mary, University of London

\subsection{6/heartjnl-2019-BCS.120}

Introduction Diagnostic genetic testing is an integral part of patient management in hypertrophic cardiomyopathy (HCM). Over time, the number of genes included in diagnostic genetic panels increased. The aim of the systematic review was to examine the impact of panel size on diagnostic yield.

Methods All cohort studies reporting the results of genetic testing in HCM patients were included, irrespective of setting. PubMed/Medline was searched from May 2000 (advent of next generation sequencing technology) to May 2018 using the following terms: (Hypertrophic Cardiomyopathy OR Hypertrophic Obstructive cardiomyopathy OR HCM OR HOCM) AND (Genotype OR Genetic Testing OR HCM panel OR pathogenic OR variant of unknown significance $O R$ VUS OR Next Generation Sequencing OR Gene* Test* OR Genotyp*). The systematic review was carried out in accordance to the Preferred Reporting Items for Systematic reviews and Meta-Analyses amendment (PRISMA).

Results A total of 4837 articles fulfilled the search criteria in PubMed and 55 observational studies were included in the systematic review. Most study cohorts were derived from European countries (47\%) and involved a single centre (73\%). Prospective data collection was employed in 32 (58\%) studies. The median cohort size was 124 patients (IQR 70-299). At least five studies included individuals $\leq 16$ years of age (the minimum age was not reported in 47 (85\%) studies).

The studies tested 2 to 15 sarcomeric protein genes (median 8 genes; IQR: 5-10), including MYH7, MYBPC3,
TNNT2, TNNI3, MYL2, MYL3, ACTC1, TPM1, TNNC1, MYH6, CSRP3, DES, TCAP, PDLIM3, PLN. All studies tested for mutations in MYBPC3 and MYH7 and most studies additionally included at least one of the following genes: TNNT2, TNNI3, MYL2, MYL3, ACTC1 and TPM1.

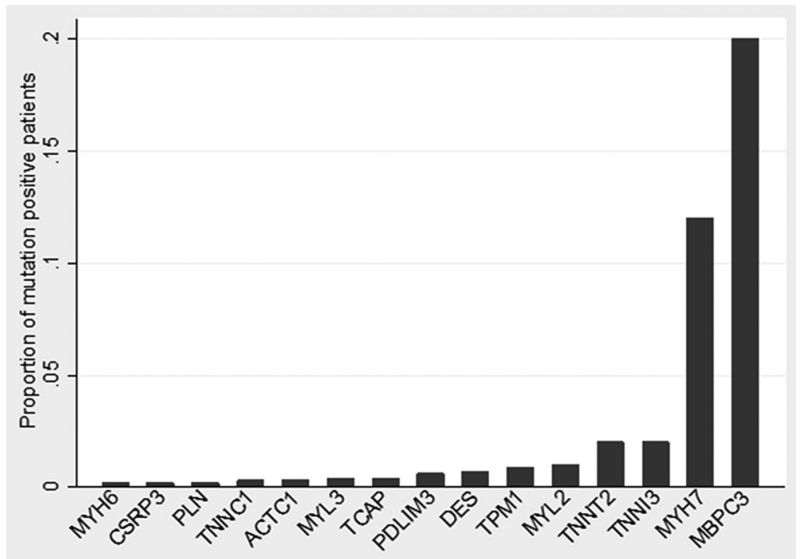

Abstract 123 Figure 1 Proportion of mutation positive patients relative to each sarcomeric gene

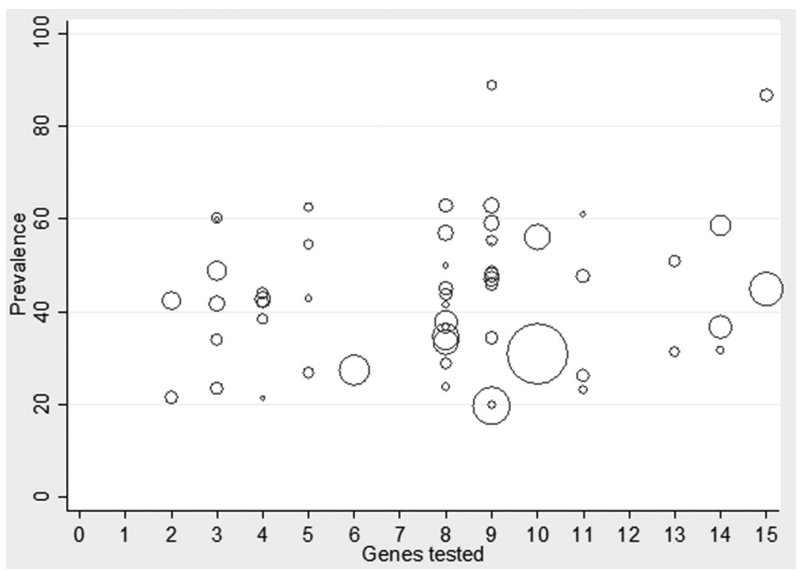

Abstract 123 Figure 2 Effect of increased genetic panel size on yield of genetic testing

NB - circle size represents size of cohort (n) 
The majority of positive mutations identified were in MYBPC3 (20\%) and MYH7 (12\%) (see figure 1). For all other sarcomeric genes, yield was $<3 \%$. Percentage yield varied greatly between studies with a range of $19-90 \%$ (mean $44 \%)$ and showed no discernible relationship with panel size (see figure 2).

Conclusion Over time, the number of genes included on HCM diagnostic genetic panels has increased but this has not translated to a significant improvement in diagnostic yield. Larger panels may introduce more complexity and uncertainty in the clinical setting and some of the high diagnostic yields may be explained by lax variant interpretation methods.

Conflict of Interest none

\section{SURVIVAL WITH VALVULAR HEART DISEASE (OXVALVE- SURVIVE)}

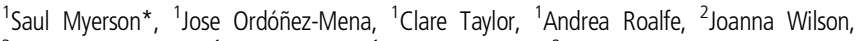
${ }^{3}$ Bernard Prendergast, ${ }^{1}$ Richard Hobbs. ${ }^{1}$ Oxford University; ${ }^{2}$ Oxford University Hospitals NHS Trust; ${ }^{3}$ St Thomas Hospital NHS Trust

10.1136/heartjnl-2019-BCS.121

Introduction Valvular heart disease (VHD) occurs commonly in older patients (>65 years) but the majority is mild disease, which is of uncertain importance. Understanding the impact of VHD on mortality in this older group of patients would help determine its relevance and aid the appropriate use of healthcare resources. We determined survival in the OxValve cohort study to investigate whether people with VHD are at increased risk of death.

Methods OxValve is a cohort study in Oxfordshire, involving systematic screening of people aged 65 and over for VHD. Over 4,009 participants were recruited between August 2009 and May 2016 and screened using echocardiography to establish the presence and severity of VHD. The cohort was linked to Office for National Statistics mortality data to obtain date and cause of death. Cox regression was used to investigate the association of any VHD, VHD of significant severity, and VHD subtypes with allcause and cause-specific mortality, adjusting for potential confounders including age, sex, socioeconomic status, smoking, and comorbidities.

Results Linked mortality data was available for 3,511 OxValve participants up to September 2018 (median 5.85 years followup). VHD was present in 2,645 (75.3\%) participants and of these $288(8.2 \%)$ had significant (moderate or severe) VHD. In total, $311(8.9 \%)$ participants had died. Cancer was the commonest cause of death $(n=135)$, followed by cardiovascular disease $(n=75)$ and respiratory disease $(n=35)$. After adjustment for age and other covariates, mild VHD was not associated with increased all-cause mortality (HR 1.16, 95\% CI: 0.89 to 1.50$)$. However, VHD of significant severity (moderate or severe disease) was associated with a nearly twofold higher risk of death overall (HR 1.92, 95\%CI: 1:38 to 2.67) including increased CVD mortality (HR 2.25, 95\% CI: 1.21 to 4.18 ) - see figure.

Conclusion Mild VHD is very common, but is not associated with increased mortality. Significant (moderate-severe) VHD was however associated with a two-fold reduction in survival. Further research is required to understand the natural history of VHD, how to identify those with progressive disease and when to intervene.

Conflict of Interest None
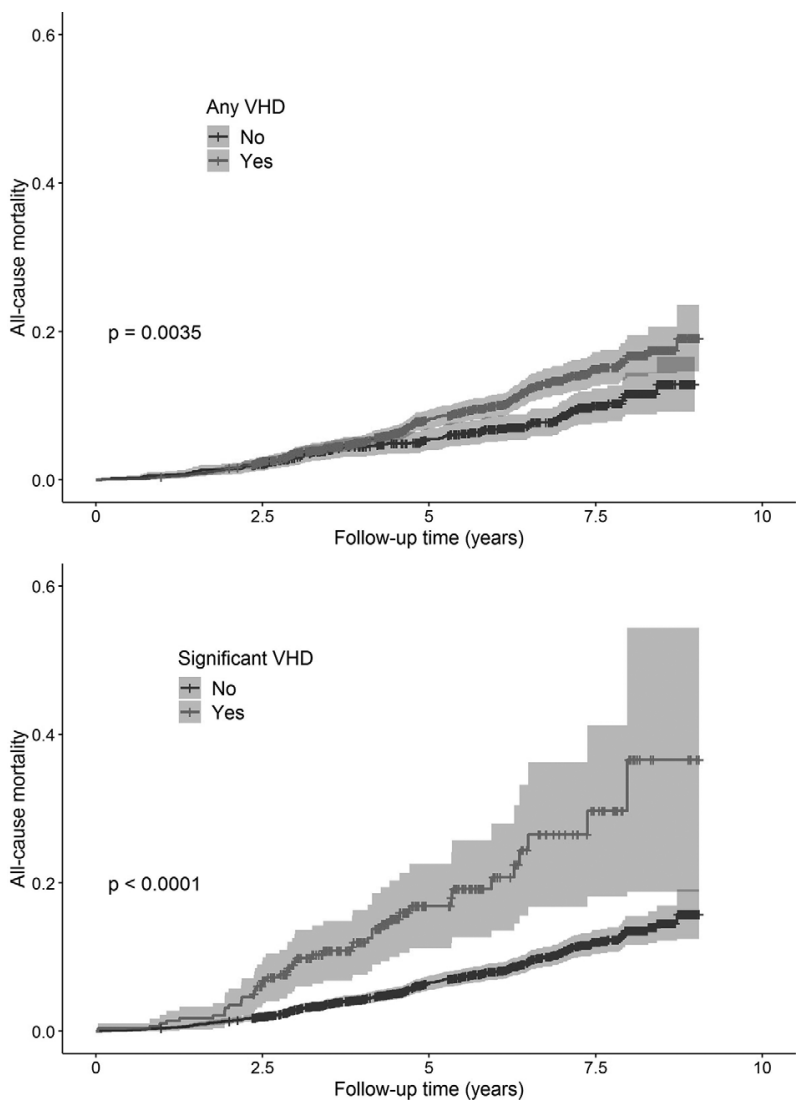

Abstract 124 Figure 1

\section{DEFINITIVE HISTOLOGICAL EVIDENCE OF MYOCARDIAL FIBROSIS IN PRIMARY DEGENERATIVE MITRAL REGURGITATION: CAUSATION OF SYMPTOMS?}

${ }^{1}$ Boyang Liu*, ${ }^{2}$ Desley Neil, ${ }^{2}$ Monisha Premchand, ${ }^{2}$ Moninder Bhabra, ${ }^{3}$ Ramesh Patel, ${ }^{3}$ Thomas Barker, ${ }^{4}$ Stephen Billing, ${ }^{4}$ Nicolas Nikolaidis, ${ }^{1}$ Nicola Edwards, ${ }^{2}$ Rick Steeds. ${ }^{1}$ University of Birmingham; ${ }^{2}$ University Hospital Birmingham; ${ }^{3}$ University Hospital Coventry; ${ }^{4}$ New Cross Hospital

\subsection{6/heartjnl-2019-BCS.122}

Chronic primary mitral regurgitation (MR) exposes the left ventricle (LV) with a volume load that leads to progressive compensatory adjustments; initially LV enlargement and eccentric hypertrophy to progressive structural and functional damage. Identifying the deleterious stages of LV remodelling before the onset of irreversible dysfunction is critical to patient management and prognosis. Myocardial fibrosis with $\mathrm{MR}$ is proposed to develop in response to increased wall stress, but data are limited to small echo based imaging biomarkers. The aim of this prospective multi-centre study is to characterise the histological status of patients with chronic primary MR and examine associated changes in cardiac structure, function and symptom burden. 120 patients with primary degenerative MR (67\% asymptomatic) were recruited prospectively. Coronary disease was excluded by angiography. All subjects underwent clinical assessment, multiparametric cardiac magnetic resonance (CMR) imaging, symptom assessment with the Minnesota Living with Heart Failure Questionnaire (MLHFQ) and cardiopulmonary exercise testing (CPET). 106 patients were referred for mitral valve surgery with the aim of collecting $3 \mathrm{LV}$ biopsies per patient. Control myocardial 